\title{
Optimal Design of Medium-and-low Speed Maglev Train Levitation Sensor for Small Radius Curve
}

\author{
Jun $\mathrm{Li}^{\mathrm{a})}$, Jun $\mathrm{Wu}^{\mathrm{b})}$ and Zhongxiu $\mathrm{Li}^{\mathrm{c})}$ \\ College of Intelligence Science and Engineering, National University of Defense Technology, Changsha 410073, \\ China. \\ a)NUDT_lijun2016@163.com, ${ }^{\text {b) }}$ wujun2008@nudt.edu.cn, ${ }^{\mathrm{c})}$ zx_nudt@126.com
}

\begin{abstract}
One of the most important features of medium-and-low speed maglev train is the ability to turn the small curve. When the train is in the small curve, as each levitation electromagnet module is a rigid structure, which cannot bend and deform, the electromagnet module and the F-shaped rail dislocate from each other, the levitation sensor installed on the end of the electromagnet module may also be offset from the center line of the F-shaped rail, which is more obvious especially on the curve of the smallest radius of $50 \mathrm{~m}$. If no measures are taken, the levitation sensor cannot provide accurate levitation gap. In the case that the levitation sensor deviates from the centerline of the F rail by $30 \mathrm{~mm}$, a mathematical model between the inductance of detecting coil and levitation gap is established, which provides an electromagnetic analysis method for $\mathrm{F}$ rail as the measured conductor and is used to optimize the parameters of the detection coil. Through the test, the results show that the levitation sensor can provide accurate levitation gap, which satisfies the requirement of levitation system, when the maglev train passes curves with $50 \mathrm{~m}$ radius.
\end{abstract}

Key words: Medium-and-Low Speed Maglev Train, Levitation Sensor, Detecting coil, Small Radius Curve, Dislocation, Magnetic Field Image Method.

\section{INTRODUCTION}

The medium-and-low speed maglev train is a new type of urban rail vehicle. It uses non-contact electromagnetic levitation and linear synchronous motor traction technology, which has the characteristics of strong hill-climbing ability, low noise and no mechanical wear [1]. The track line can be flexibly planned according to the conditions of the city's ground buildings, and the minimum turning radius can reach 50m. As shown in Figure 1, the levitation gap is defined as the distance between the polar face of the electromagnet and the lower surface of the track. When the gap between the pole face of the electromagnet and the lower pole surface of the track is tight, the levitation gap is defined as $0 \mathrm{~mm}$. In order to avoid collision between the levitation sensor and the F-shaped rail (abbreviated as F rail) concave surface, there is a gap of $4 \mathrm{~mm}$ between the detection coil and the detection surface. Therefore, the levitation sensor defines a levitation gap of $0-20 \mathrm{~mm}$, the actual detection gap is $4-24 \mathrm{~mm}$. During the operation of the maglev vehicle, the levitation control system maintains the levitation gap at about $8 \mathrm{~mm}$, the levitation sensor is located at the end of the electromagnet, and the detection coil is at the concave surface of the F rail, used to detect the levitation gap. At the same time, the vertical acceleration of the suspension electromagnet is provided. Because the eddy current sensor has the characteristics of non-contact measurement and wide dynamic response range, and it is suitable for detecting gaps of a few millimeters to tens of millimeters, So the levitation sensor adopts the eddy current form [2], and the vertical acceleration measurement is implemented by using an accelerometer inside. This paper focuses on the detection of levitation gap for a vehicle on a small radius curve. 


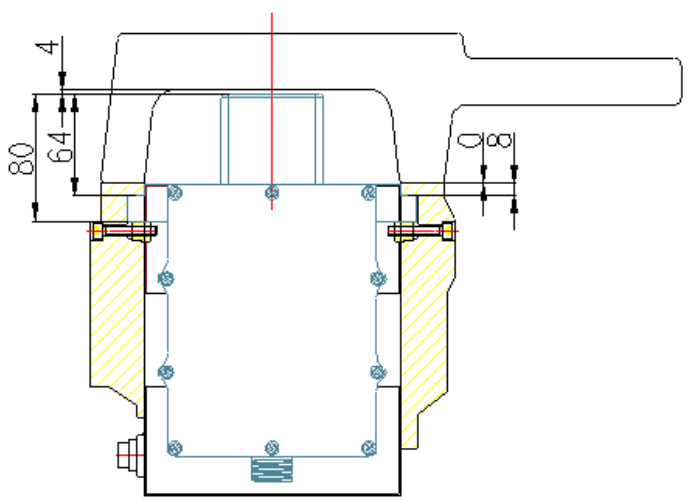

(a)Definition of $0 \mathrm{~mm}$ levitation gap

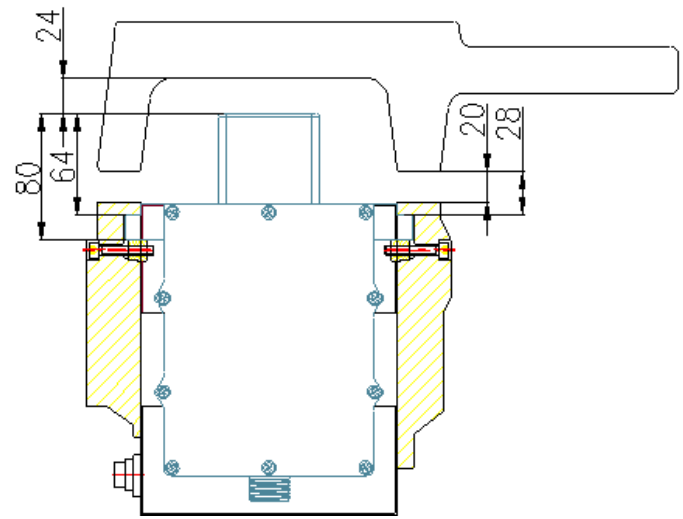

(b)Definition of $20 \mathrm{~mm}$ levitation gap

FIG. 1. Definition of 0-20mm levitation gap

The detection surface of the levitation sensor is the plane of the concave surface of the F rail, and the width is about $110 \mathrm{~mm}$. The high-frequency detection magnetic field of the detection coil is an open magnetic field. In order to realize the range of $24 \mathrm{~mm}$, the outer diameter of the detection coil must be designed to be large enough. Generally, the outer diameter of the coil is 2-3 times that of the measuring range [3]. Therefore, the levitation sensor needs to use the detection coil which has a larger outer diameter. When the train is running on a straight section, the detection coil of the levitation sensor substantially coincides with the centerline of the F rail. Although the detection magnetic field is an open magnetic field, the detection plane is wide enough, the side edge of the $\mathrm{F}$ rail has little effect on the detection magnetic field and does not affect the detection of the gap. When the train passes a small curve, especially a $50 \mathrm{~m}$ curve radius, the electromagnet module is misaligned with the centerline of the $\mathrm{F}$ rail. The detection coil of the levitation sensor will be very close to the edge of the F rail. If no measures are taken, the side edge of the $\mathrm{F}$ rail will affect the open detection magnetic field, resulting in inaccurate clearance signals and affecting the normal levitation of the train. If the train wants to levitate on the curve from the $17 \mathrm{~mm}$ levitation gap, the dislocation will have a more pronounced effect on the gap detection, and the possibility of instability or landing of the levitation system is greater, which will determine the train's ability to pass through curves. Therefore, it is very meaningful to study the levitation sensor to adapt to the curve of small radius.

To solve the above problems, the design and optimization based on the mathematical model of the sensor detection coil is the main solution. In recent years, some theoretical calculation models have been proposed for the impedance calculation of planar inductive coils. Acero proposed an electromagnetic field vector calculation model for circular planar coils [4]. Fava established a second-order impedance vector model for an arbitrary-rotation square spiral planar coil [5]. Tröltzsch U used magnetic vector potential theory to establish a mathematically simple coil self-capacitance and internal inductance model [6]. Vogel $\mathrm{J}$ studied the inductance analysis model of eddy current sensor at high excitation frequency [7]. Because of the particularity of the application environment, except for some patents currently involved, there is the no method for calculation of the equivalent inductance of the levitation sensor detection coil. For the analysis and design of the detection coils, the existing literature proposed the equivalent inductance calculation model under the assumption that the detection plane is infinitely large. The problem faced by levitation sensor is not only that the measured plane is the lower surface of the finite width of the $\mathrm{F}$ rail, but also that in the case of a large dislocation, the side edge of the $\mathrm{F}$ rail will affect the detection magnetic field. The measuring surface at this time has not satisfied the condition of infinite plane.

Based on the theory of magnetic field image method, in the case that the levitation sensor deviates from the centerline of the $\mathrm{F}$ rail by $30 \mathrm{~mm}$, this paper analyzes the influence of the $\mathrm{F}$ rail side edge on the gap detection according to the characteristics of the circular inductor coil. A mathematical model between the inductance of detecting coil and levitation gap is established, which provides an electromagnetic analysis method for $\mathrm{F}$ rail as the measured conductor. The model is used to optimize the parameters of the detection coil and improves the adaptability of the levitation sensor to the curve of small radius. 


\section{THE DISLOCATION BETWEEN THE LEVITATION SENSOR AND F RAIL AT THE CURVE}

As shown in Fig. 2, one carriage of medium-and-low speed maglev train consists of five trucks, two levitation electromagnet modules are installed at the bottom of each truck, each levitation electromagnet module has two levitation sensors installed. The levitation sensor's detection coils are located in a narrow space between the concave surface of the $\mathrm{F}$ rail and the electromagnet module. Figure 3 shows the structure of the levitation sensor. It provides three independent gap signals and two acceleration signals. The three detection coils are equally distributed on the cantilever of the sensor. The detection range of the levitation sensor is $0-20 \mathrm{~mm}$.

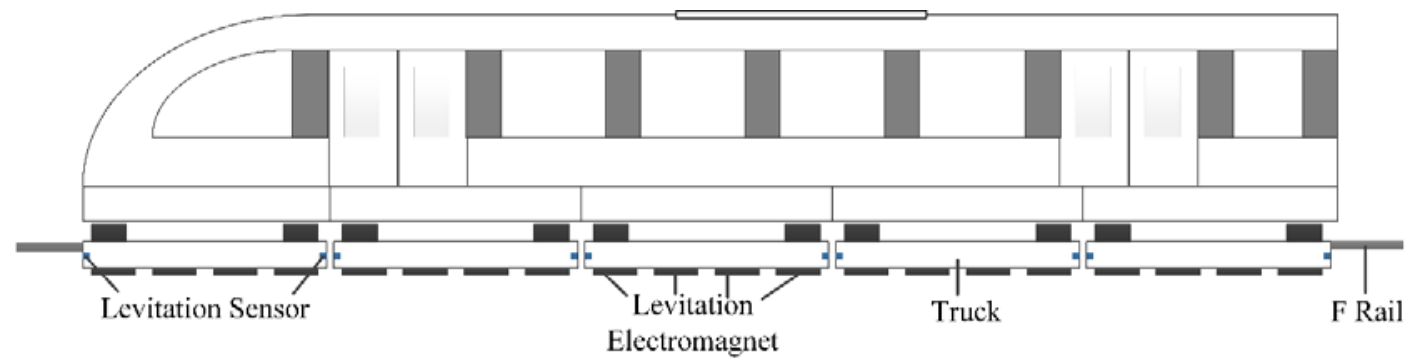

FIG. 2. The position distribution of levitation sensor

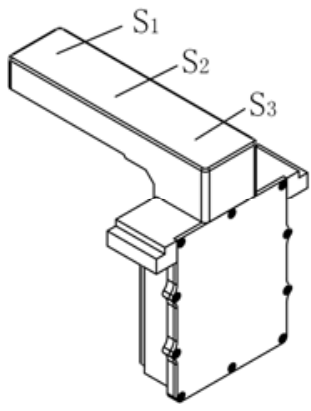

FIG. 3. The structure of the levitation sensor

One carriage of medium-and-low speed maglev train is about $15.5 \mathrm{~m}$, the length of single truck is about $2.72 \mathrm{~m}$, the maximum length is less than $4 \mathrm{~m}$. Levitation control system requires that the maglev train has the ability to stably pass through curves with $50 \mathrm{~m}$ radius and stably levitate at curves with $50 \mathrm{~m}$ radius. According to the requirements of the control system, the levitation control system allows the maximum detection error of the sensor to be $0.5 \mathrm{~mm}$ under that condition.

When the train is at curves with $50 \mathrm{~m}$ radius, the dislocation between the detection coil and the $\mathrm{F}$ rail is largest, and the offset $\Delta$ is calculated according to the following equation [8]:

$$
\Delta \approx \frac{l_{m}^{2}}{16 R}
$$

Where $l_{\mathrm{m}}$ is the length of the truck, $\mathrm{R}$ is the radius of curve.

The offset $\Delta \leq \frac{4^{2}}{16 \cdot 50}=0.02 \mathrm{~m}$. In order to ensure that the levitation sensor can effectively adapt to the curve of small radius, the design and analysis of the detection coil is performed under the condition that the maximum misalignment offset is $30 \mathrm{~mm}$. 


\section{PRELIMINARY DESIGN FOR DETECTION COIL AND ANALYSIS OF MAGNETIC FIELD IMAGE METHOD}

Several boundary constraints or assumptions of the detection coil are to be analyzed. Firstly, the sensor uses a multi-detection coil form, which has three independent detection coils. In order to prevent influence between the detection coils, different detection coils adopt different excitation frequencies and the narrowband filter is designed. Therefore, the interaction between the redundantly designed coils can be ignored, the model analysis in this paper is just only for one detection coil. Secondly, the actual detection range of the levitation sensor is 4-24mm. To meet the requirements, the preliminary design of the detection coil is a planar circular inductor with a diameter of $40 \mathrm{~mm}$, a number of turns of 150, and a thickness of $3 \mathrm{~mm}$. Finally, ignore the effects of external magnetic field on the coil, such as levitation magnetic fields. Therefore, the analysis and calculation model can be simplified to the form of only the F rail and the detection coil, when the train is normally levitated at the curve.

In the case of deviating from the centerline of the $\mathrm{F}$ rail by $30 \mathrm{~mm}$, the gap detection deviation is shown in Table 1 , and it can be seen that the error is larger at the end of the sensor range, especially when the levitation gap is $18 \mathrm{~mm}$, the error is $0.738 \mathrm{~mm}$, which does not satisfy the error required for a levitation system to be less than $0.5 \mathrm{~mm}$.

TABLE 1. Levitation gap error under initial coil parameters

\begin{tabular}{cccccccc}
\hline Levitation & $\Delta / \mathrm{mm}$ & $\begin{array}{c}\text { Levitation } \\
\text { Gap } / \mathrm{mm}\end{array}$ & $\Delta / \mathrm{mm}$ & $\begin{array}{c}\text { Levitation } \\
\text { Gap } / \mathrm{mm}\end{array}$ & $\Delta / \mathrm{mm}$ & \multicolumn{2}{c}{ Levitation } \\
Gap $/ \mathrm{mm}$ & $\Delta / \mathrm{mm}$ \\
\hline 1 & 0.049 & 6 & 0.016 & 11 & 0.092 & 16 & 0.329 \\
2 & -0.003 & 7 & 0.001 & 12 & -0.088 & 17 & 0.532 \\
3 & -0.029 & 8 & 0.061 & 13 & 0.201 & 18 & 0.738 \\
4 & 0.023 & 9 & 0.083 & 14 & 0.109 & 19 & 0.4983 \\
5 & 0.054 & 10 & 0.071 & 15 & 0.156 & 20 & 0.5308 \\
\hline
\end{tabular}

\section{Analysis of Magnetic Field of Circular Coil.}

Let the radius of the circular coil be R, the number of turns is N, and the current is I. As shown in Figure 4, establish a Cartesian coordinate system with the center of the circle as the origin.

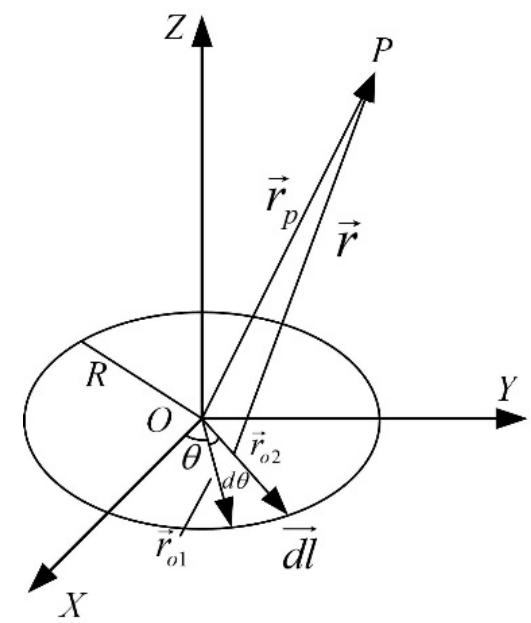

FIG. 4. Magnetic field calculation of circular coil

The formula for calculating the magnetic field at any point around the circular coil is as follow. 


$$
\begin{gathered}
\vec{B}(x, y, z)=B_{x} \vec{i}+B_{y} \vec{j}+B_{z} \vec{k} \\
=\frac{\mu_{0} N I R}{4 \pi}\left[z B_{1 x} \vec{i}+z B_{1 y} \vec{j}+\left(B_{1 z}-x B_{1 x}-y B_{1 y}\right) \vec{k}\right] \\
B_{x}=\frac{\mu_{0} N I R z}{4 \pi} B_{1 x}, B_{y}=\frac{\mu_{0} N I R z}{4 \pi} B_{1 y}, B_{z}=\frac{\mu_{0} N I R}{4 \pi}\left(B_{1 z}-x B_{1 x}-y B_{1 y}\right) \\
B_{1 x}=\int_{0}^{2 \pi} \frac{\cos \theta d \theta}{\left[(x-R \cos \theta)^{2}+(y-R \sin \theta)^{2}+z^{2}\right]^{3 / 2}} \\
B_{1 y}=\int_{0}^{2 \pi} \frac{\sin \theta d \theta}{\left[(x-R \cos \theta)^{2}+(y-R \sin \theta)^{2}+z^{2}\right]^{3 / 2}} \\
B_{1 z}=\int_{0}^{2 \pi} \frac{R d \theta}{\left[(x-R \cos \theta)^{2}+(y-R \sin \theta)^{2}+z^{2}\right]^{3 / 2}}
\end{gathered}
$$

When the eddy current sensor is in no-load condition, the magnetic flux of per coil is

$$
\Phi=\iint_{S} \vec{B} \cdot \vec{n} d S
$$

Where $\vec{B}$ is magnetic induction intensity vector around the detection coil, such as Equation 2; S usually is the area of the coil. $\vec{n}$ is the normal unit vector of coil plane.

Then the no-load equivalent inductance of the detection coil is:

$$
L=\frac{N}{I} \iint_{S} \vec{B} \cdot \vec{n} d S
$$

\section{Electromagnetic Calculation Between Detection Coil and F Rail.}

The difficulty in electromagnetic calculation between the detection coil and the F rail lies in the constraints of the boundary conditions between the two. If the constraint can be ignored, the coil equivalent inductance calculation is the same as the calculation of the no-load coil inductance. The Magnetic Field Image Method can solve the magnetic field of the two media equivalently as a media magnetic field, there are two equivalent conditions: the edge effect of the interface is negligible, and the shape of the interface satisfies the acute angle condition [9].

When the gap is small, A3 steel can meet these conditions, so the paper use the magnetic field mirror method to solve the magnetic field distribution of the circular coil, calculate the equivalent inductance of the detection coil.

According to the magnetic field image method, considering the structure of the F rail and the characteristics of the A3 steel, the model between the detection coil and the A3 steel is shown in Fig. 5(b), in which two planes form an angular boundary. Outside the angular field, there are three mirrored coils (L1, L2 and L3), which are identical to the detection coil L. If the excitation current of the detection coil is $\mathrm{I}$, the mirror current is $\mathrm{I} 1=\mathrm{I}$. The magnetic field in this angular domain is formed by the superposition of the magnetic fields generated by these four coils. A rectangular coordinate system o-xyz is established, and the z-axis is parallel to the central axis of the circular coil. To describe the symmetry of the two coils, the xoy plane and the yoz plane are the interfaces between air and A3 
steel. $\Omega$ is the solution domain: $\mathrm{z}<0 . \mathrm{d} 1$ is the gap between the coil and the A3 steel. $\mathrm{d} 2$ is the distance between the coil and the side edge of the $\mathrm{F}$ rail. $\mathrm{ra}$ is the outer diameter and $\mathrm{rb}$ is the inner diameter of a circular coil. The unit vectors in the Cartesian coordinate system $\mathrm{x}, \mathrm{y}$, and $\mathrm{z}$ directions are $\vec{i}, \vec{j}, \vec{Z}$.

After the mirror coil is placed, the entire coordinate space of the equivalent model is only air. In this way, the magnetic induction of L, L1, L2 and L3 can be calculated separately. The magnetic field superposition principle is used to obtain the magnetic induction of the detection coil, and then obtain the equivalent inductance-gap equation.

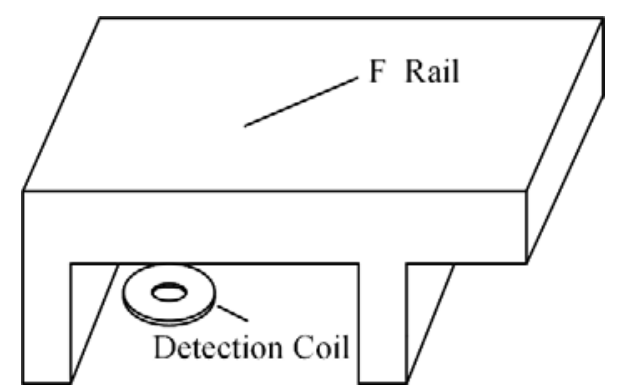

(a)Detection coil and $\mathrm{F}$ rail

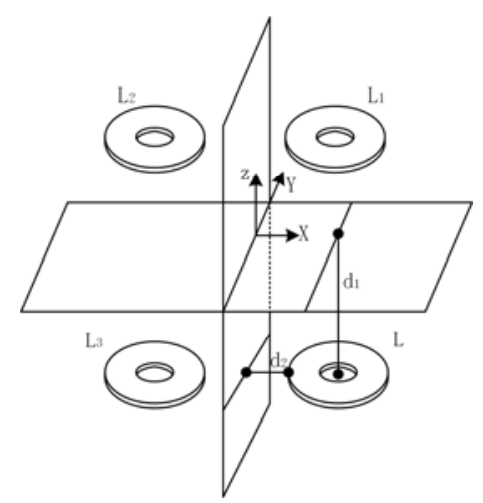

(b) Equivalent calculation model

FIG. 5. Calculation Model Based on Magnetic Field Image Method

Comparing Fig. 4 and Fig. 5(b), we can see that the difference between the two is that the coordinate system has been translated. Calculate the magnetic induction intensity distributions of L, L1, L2 and L3 according to Equation 2 and the coordinate transformation between the two coordinates, the magnetic induction intensity in the $\Omega$ domain is obtained after superposition.

The magnetic induction intensity of $\mathrm{L}, \mathrm{L} 1, \mathrm{~L} 2$ and $\mathrm{L} 3$ at the detection coil are $\vec{B}_{\delta 0}(x, y, z), \vec{B}_{\delta 1}(x, y, z)$,

$\vec{B}_{\delta 2}(x, y, z)$ and $\vec{B}_{\delta 3}(x, y, z)$.

$$
\left\{\begin{array}{l}
\vec{B}_{\delta 0}(x, y, z)=\vec{B}\left(x-d_{2}-r_{a}, y, z+d_{1}\right) \\
\vec{B}_{\delta 1}(x, y, z)=\vec{B}\left(x-d_{2}-r_{a}, y, z-d_{1}\right) \\
\vec{B}_{\delta 2}(x, y, z)=\vec{B}\left(x+d_{2}+r_{a}, y, z-d_{1}\right) \\
\vec{B}_{\delta 3}(x, y, z)=\vec{B}\left(x+d_{2}+r_{a}, y, z+d_{1}\right)
\end{array}\right.
$$

Where $B(x, y, z)$ is shown in Equation 2, the magnetic induction intensity is superimposed to obtain the magnetic induction intensity of the detection coil.

$$
\vec{B}_{\delta}\left(x, y,-d_{1}\right)=\vec{B}_{\delta 0}\left(x, y,-d_{1}\right)+\vec{B}_{\delta 1}\left(x, y,-d_{1}\right)+\vec{B}_{\delta 2}\left(x, y,-d_{1}\right)+\vec{B}_{\delta 3}\left(x, y,-d_{1}\right)
$$

The relationship between the equivalent inductance of the detection coil and the levitation gap is 


$$
\begin{aligned}
L= & \frac{N}{I} \iint_{S} \vec{B}_{\delta}\left(x, y,-d_{1}\right) \cdot \vec{n} d S \\
= & \frac{N}{I} \iint_{S} \vec{B}\left(x-\left(d_{2}+r_{a}\right), y, 0\right) \cdot \vec{n} d S+\frac{N}{I} \iint_{S} \vec{B}\left(x-\left(d_{2}+r_{a}\right), y,-2 d_{1}\right) \cdot \vec{n} d S+ \\
& \frac{N}{I} \iint_{S} \vec{B}\left(x+\left(d_{2}+r_{a}\right), y,-2 d_{1}\right) \cdot \vec{n} d S+\frac{N}{I} \iint_{S} \vec{B}\left(x+\left(d_{2}+r_{a}\right), y, 0\right) \cdot \vec{n} d S
\end{aligned}
$$

In the equation, the first item is the static self-inductance of the detection coil, and the second item is the mutual inductance between the detection coil and the lower surface of $\mathrm{F}$ rail. Let L0 be the sum of these two items. It can be assumed that L0 is the inductance of the coil without deviating from the centerline of the F rail. The remaining two items are the mutual inductance L1 between the detection coil and the side edge of the F rail. It can be considered that the smaller L1 is, the smaller the influence of the side edge of the F rail on the levitation sensor.

$$
\begin{aligned}
& L_{0}=\frac{N}{I} \iint_{S} \vec{B}\left(x-\left(d_{2}+r_{a}\right), y, 0\right) \cdot \vec{n} d S+\frac{N}{I} \iint_{S} \vec{B}\left(x-\left(d_{2}+r_{a}\right), y,-2 d_{1}\right) \cdot \vec{n} d S \\
& L_{1}=\frac{N}{I} \iint_{S} \vec{B}\left(x+\left(d_{2}+r_{a}\right), y,-2 d_{1}\right) \cdot \vec{n} d S+\frac{N}{I} \iint_{S} \vec{B}\left(x+\left(d_{2}+r_{a}\right), y, 0\right) \cdot \vec{n} d S
\end{aligned}
$$

Calculated by Equation 1 and 11, under the condition that the levitation sensor and the F rail is dislocated, it is necessary to reduce the number of turns of the coil, the outer diameter of the coil, the inner diameter and the thickness, and the detection coil should be in the form of a small outer diameter and a thin coil, so that the side edge of the F rail has the least influence on the detection coil and the gap signal can be accurate. However, in order to ensure the sensor has a suitable range, the outer diameter of the detection coil cannot be too small; in order to achieve a higher sensitivity of the sensor, the thickness of the detection coil should be thin. Referring to the experimental test, adjust the coil design to a diameter of $36 \mathrm{~mm}$, a number of turns of 100 , and a thickness of $1 \mathrm{~mm}$ circular inductor coil.

\section{FINITE ELEMENT SIMULATION ANALYSIS AND TEST}

In order to verify whether the calculation equation is correct or not, it is a way to compare the simulation results with the calculation results. The finite element calculation is a common simulation calculation method.

\section{Simulation Analysis of Magnetic Induction of Detection Coil.}

According to the adjusted parameters of the detection coil, the Maxwell 3D was used to simulate and calculate the electromagnetic field. Enter the geometric model and physical parameters. The diameter of the circular coil is $36 \mathrm{~mm}$, the thickness is $1 \mathrm{~mm}$, the number of turns is 100 , and the excitation current $\mathrm{I}$ is $0.3 \mathrm{~A}$. The coil is parallel to the plane of the F rail.

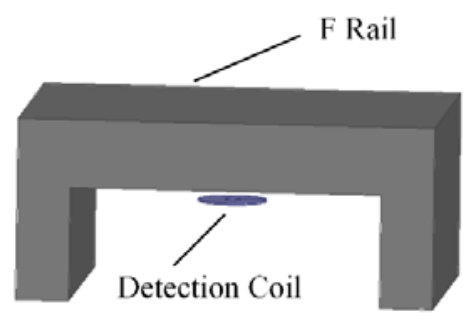

FIG. 6. Simulation model 
In the case of deviation from the centerline of the $\mathrm{F}$ rail by $30 \mathrm{~mm}$, the inductance of the detection coil under the $0-20 \mathrm{~mm}$ levitation gap was calculated by simulation, and the equivalent inductance-gap curve was obtained as shown in Figure 7.

Compared with the finite element simulation and calculation results, the distribution rule of the inductance is consistent: The electromagnetic calculation based on the magnetic field image method is reasonable.

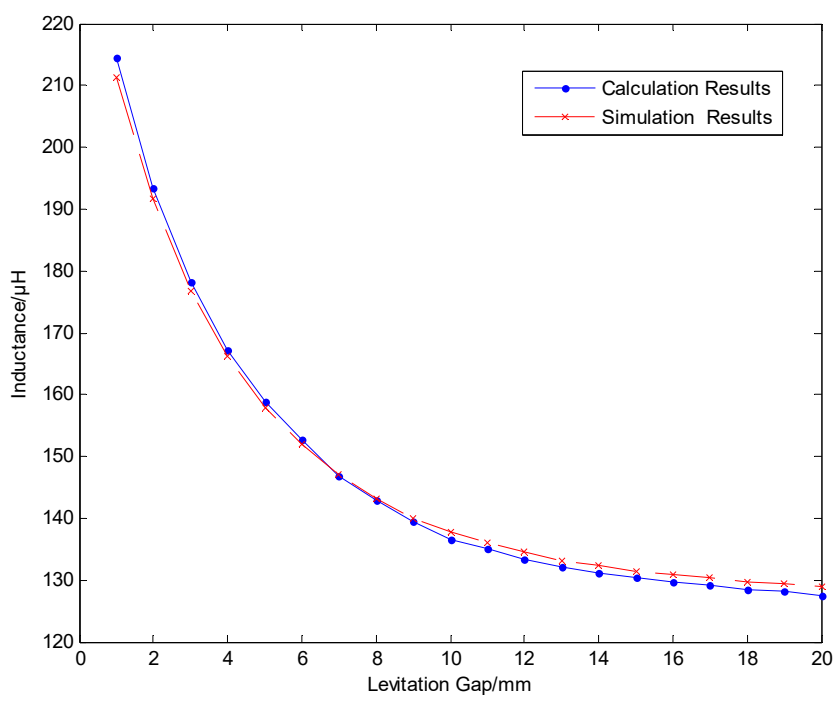

FIG. 7. Comparison of simulation results and calculation results

\section{Experimental Verification.}

After the coil parameters were readjusted, in the case of deviation from the centerline of the F rail by $30 \mathrm{~mm}$, the gap detection deviation was as shown in Table 2. It can be seen that after the parameter adjustment, it is less than $0.3 \mathrm{~mm}$ in the range of $0-20 \mathrm{~mm}$, which can meet the requirement of the levitation control system.

TABLE 2. Levitation gap error under readjusted coil parameters

\begin{tabular}{|c|c|c|c|c|c|c|c|}
\hline $\begin{array}{l}\text { Levitation } \\
\text { Gap /mm }\end{array}$ & $\Delta / \mathrm{mm}$ & $\begin{array}{l}\text { Levitation } \\
\text { Gap /mm }\end{array}$ & $\Delta / m m$ & $\begin{array}{l}\text { Levitation } \\
\text { Gap /mm }\end{array}$ & $\Delta / m m$ & $\begin{array}{l}\text { Levitation } \\
\text { Gap /mm }\end{array}$ & $\Delta / \mathrm{mm}$ \\
\hline 1 & 0.184 & 6 & 0.071 & 11 & -0.023 & 16 & -0.266 \\
\hline 2 & 0.011 & 7 & 0.051 & 12 & 0.242 & 17 & 0.051 \\
\hline 3 & -0.027 & 8 & 0.054 & 13 & 0.013 & 18 & -0.085 \\
\hline 4 & 0.007 & 9 & -0.012 & 14 & -0.189 & 19 & -0.075 \\
\hline 5 & 0.027 & 10 & 0.087 & 15 & -0.027 & 20 & 0.251 \\
\hline
\end{tabular}

At the same time, When the readjusted detection coil of the levitation sensor substantially coincides with the centerline of the F rail, the output gap signal is accurate, which can meet the requirement of the levitation control system.

Experiments show that the redesigned levitation sensor can meet the requirements of the levitation system.

\section{SUMMARY}

Based on the theory of magnetic field image method, the paper designs and analyzes the levitation sensor's detection coil for the levitation sensor structure and specialty, the vehicle system's requirements for the sensor.

1. In the case of deviation from the centerline of the $\mathrm{F}$ rail, a mathematical model of the detection coil inductance - levitation gap is established. Comparing the results of finite element simulation and mathematical 
model calculations, the distribution rule of the detection coil inductance is generally consistent. It is considered that the electromagnetic calculation model of the coil based on the magnetic field image method is reasonable. The paper provides an electromagnetic analysis method for the $\mathrm{F}$ rail.

2. Based on the analysis results of the electromagnetic calculation model, the detection coil parameters were adjusted. The use of a thin coil with a small outer radius can effectively avoid the influence of the side edge of the $\mathrm{F}$ rail on the levitation sensor. Through experimental verification, whether the vehicle is on a straight or small curve, the levitation sensor can meet the requirements of the levitation control system in the range of $0-20 \mathrm{~mm}$.

\section{ACKNOWLEDGEMENTS}

National Key R \& D Program of China (2016YFB1200602-40).

\section{REFERENCES}

1. Wu Xiangming. Maglev Train [M]. Shanghai: Shanghai Scientific \& Technical Publishers, 2003:1-5.

2. Song Qiang, Zhang Ye, Wang Rui. Sensor principle and Application Technology [M]. Chengdou: Southwest Jiaotong University Press,2016:98-128.

3. Yu Yating, Du pingan, Liao yaqin. study on effect of coil shape and geometric parameters on performance of eddy current sensor[J]. Chinese Journal of scientific Instrument,2007,28(06):1045-1050.

4. Acero J. Alonso R. Barragan L A, et al. Magnetic vector potential based model for eddy-current loss calculation in round-wire planar windings [J]. IEEE Transactions on Magnetics, 2006,42(9):2152-2158.

5. Fava J O, Ruch M C. Calculation and simulation of impedance diagrams of planar rectangular spiralcoils for eddy current testing [J]. NDT \& E international, 2006,39(5):414-424.

6. Tröltzsch U, Wendler F, Kanoun O. Simplified analytical inductance model for a single turn eddy current sensor [J]. Sensors \& Actuators A Physical, 2013, 191(3):11-21.

7. Vogel J, Nihtianov S. Modelling the inductance of a novel eddy-current position sensor for high-precision applications[C]// Sensors Applications Symposium. IEEE, 2016:1-6.

8. Li Yunfeng. Study and Design Theoretically of Walking Mechanism on Five Truck Middle and Low Speed Maglev Train [D]. Changsha: National University of Defense Technology,2007:17-19.

9. K. J. BINNS, P. J. LAWRENSON. Analysis and Computation of Electric and Magnetic Field Problems [M]. PERGAMON PRESS, 1973:26-41. 